\title{
Discovery of Secular Evolution of the Atmospheric Abundances of Ap Stars
}

\author{
J. D. Bailey ${ }^{1}$, J. D. Landstreet ${ }^{2,3}$ and S. Bagnulo ${ }^{3}$ \\ ${ }^{1}$ Max Planck Institut für extraterrestrische Physik, Giessenbachstrasse 1, 85748 Garching, \\ Germany \\ email: jeffbailey@mpe.mpg.de \\ ${ }^{2}$ Department of Physics and Astronomy, The University of Western Ontario, London, Ontario, \\ N6A 3K7, Canada \\ ${ }^{3}$ Armagh Observatory, College Hill, Armagh, BT61 9DG, Northern Ireland, UK
}

\begin{abstract}
The stars of the middle main-sequence have relatively quiescent outer layers, and unusual chemical abundance patterns may develop in their atmospheres, revealing the action of such subsurface phenomena as gravitational settling and radiatively driven levitation of trace elements, and their competition with mixing processes such as turbulent diffusion. We report the discovery of the time evolution of such chemical tracers through the main-sequence lifetime of magnetic chemically peculiar stars.
\end{abstract}

Keywords. stars: abundances, stars: chemically peculiar, stars: magnetic fields

\section{Introduction}

The A- and late B-type main sequence stars do not have any single powerful process acting to force the surface layers to retain essentially their initial chemical composition. As a result, many $\mathrm{A}$ and $\mathrm{B}$ stars show chemical abundances remarkably different from those measured in the Sun. Among A and B stars there are several families of distinctive compositional patterns that reflect different broad sets of physical conditions. The compositional peculiarities vary rather strongly with effective temperature (and thus with the momentum carried by the outflowing radiation that levitates some trace elements) and are very different, depending on whether the star has a strong magnetic field or not. These chemical peculiarities provide powerful probes of invisible processes occurring beneath the visible layers of all kinds of stars, particularly of upward and downward diffusion (Landstreet 2004). It is therefore of obvious interest to observe and characterise the importance and time-scale of these phenomena.

A major difficulty of interpreting observed chemical signatures has been that the ages of isolated (field) A and B stars can be estimated only approximately. We have dealt with this issue by performing accurate abundance analysis on a sample of high-resolution spectra of magnetic A and B stars that are open cluster members. The ages of cluster members can be determined with much better accuracy than that of individual stars, and the cluster age applies to all its members (which are presumed to have formed essentially contemporaneously) (Bagnulo et al. 2006; Landstreet et al. 2007). We have determined the chemical compositions of 15 stars with masses of $3.5 \pm 0.5 M_{\odot}$ and ages between $5 \cdot 10^{6} \mathrm{yr}$ and $2.5 \cdot 10^{8} \mathrm{yr}$. In effect, by studying the changes in average atmospheric chemical composition with cluster age, we are observing the time evolution of the chemistry of a $3.5 M_{\odot}$ star through its main sequence life. 

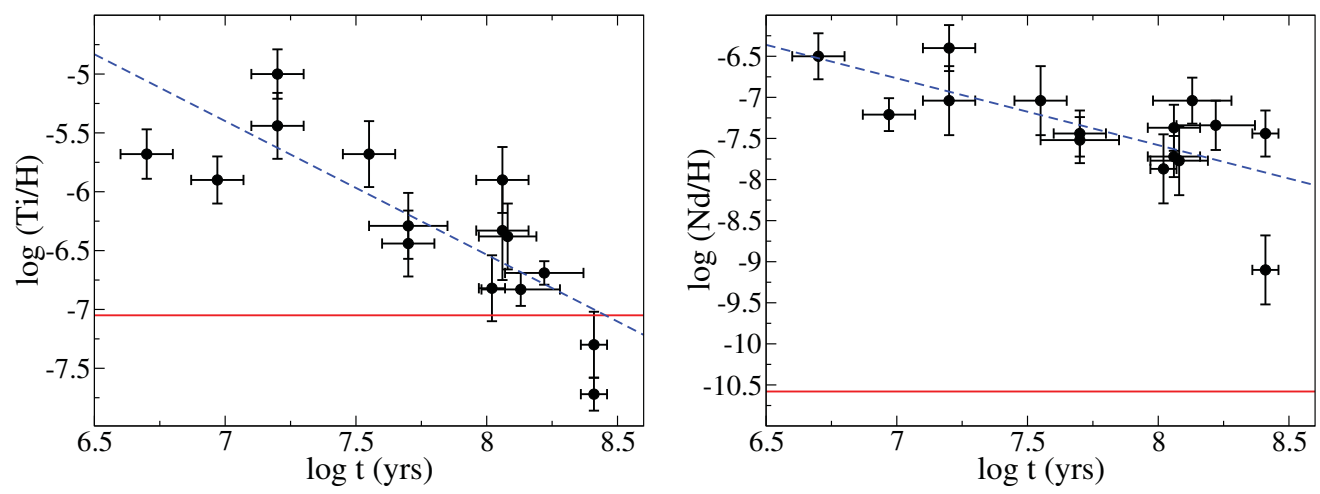

Figure 1. Observed evolution of the abundances of $\mathrm{Ti}$ and $\mathrm{Nd}$ with main sequence age for a $3.5 M_{\odot}$ star. The solar abundance ratio is plotted as a solid red line.

\section{Methods}

To model the atmospheric abundances of the magnetic Ap stars, the spectrum synthesis program ZEEMAN was used (Landstreet 1988; Wade et al. 2001). When performing the abundance analysis, a uniform atmospheric abundance distribution over the stellar surface was assumed. For each star, a simple dipolar magnetic field with the polar field value $B_{\mathrm{d}}$ approximately three time $B_{\mathrm{rms}}$ was assumed, with the line-of-sight parallel to the magnetic field axis. The microturbulence parameter was set to 0 (because the magnetic field suppresses any convective motions). When multiple spectra were available, the two that exhibit the greatest differences were analysed, and average abundance of the two modelled spectra was adopted.

\section{Results}

During the main sequence life of a $3.5 M_{\odot}$, magnetic chemically peculiar A-type (Ap) star, the abundances:

- of the light elements $(\mathrm{O}, \mathrm{Mg}, \mathrm{Si})$ remain roughly constant. Compared to the Sun, O and $\mathrm{Mg}$ are in general underabundant, whereas $\mathrm{Si}$ is always overabundant.

- of the Fe-peak (Ti, Cr, Fe) and rare-earth (Pr, Nd) elements decrease. The former evolve from large overabundances to nearly solar values. The latter exhibit drastic overabundances in young Ap stars (of the order of $10^{4}$ times greater than in the Sun) decreasing to values still about 100 times larger than the solar ratios.

- of He unexpectedly increases from about $1 \%$ to $10 \%$ of the solar He abundance. The nature of this increase is not yet understood.

Fig. 1 shows two examples of these results. We refer the reader to Bailey et al. (2014), where a complete discussion of this work is provided.

\section{References}

Bagnulo, S., Landstreet, J. D., Mason, E., et al. 2006, A\&A 450, 777

Bailey, J. D., Landstreet, J. D., \& Bagnulo, S. 2014, A\&SA 561, A147

Landstreet, J. D. 1988, ApJ 326, 967

Landstreet, J. D. 2004, in J. Zverko, J. Ziznovsky, S. J. Adelman, \& W. W. Weiss (eds.), The A-Star Puzzle, Vol. 224 of IAU Symposium, pp 423-432

Landstreet, J. D., Bagnulo, S., Andretta, V., et al. 2007, A\&A 470, 685

Wade, G. A., Bagnulo, S., Kochukhov, O., et al. 2001, A\&A 374, 265 